\title{
Non-destructive Thickness Mapping of Wafer-Scale Hexagonal Boron Nitride Down to a Monolayer
}

Crovetto, Andrea; Whelan, Patrick Rebsdorf; Wang, Ruizhi; Galbiati, Miriam; Hofmann, Stephan; Camilli, Luca

\section{Published in:}

A C S Applied Materials and Interfaces

Link to article, DOI:

10.1021/acsami.8b08609

Publication date:

2018

Document Version

Peer reviewed version

Link back to DTU Orbit

Citation (APA):

Crovetto, A., Whelan, P. R., Wang, R., Galbiati, M., Hofmann, S., \& Camilli, L. (2018). Non-destructive Thickness Mapping of Wafer-Scale Hexagonal Boron Nitride Down to a Monolayer. A C S Applied Materials and Interfaces, 10, 2580425810. https://doi.org/10.1021/acsami.8b08609

\section{General rights}

Copyright and moral rights for the publications made accessible in the public portal are retained by the authors and/or other copyright owners and it is a condition of accessing publications that users recognise and abide by the legal requirements associated with these rights.

- Users may download and print one copy of any publication from the public portal for the purpose of private study or research.

- You may not further distribute the material or use it for any profit-making activity or commercial gain

- You may freely distribute the URL identifying the publication in the public portal 


\title{
Non-Destructive Thickness Mapping of Wafer-Scale Hexagonal Boron Nitride Down to a Monolayer
}

\author{
Andrea Crovetto ${ }^{1,2 *}$, Patrick Rebsdorf Whelan ${ }^{1,3,4}$, Ruizhi Wang $^{5}$, Miriam Galbiati ${ }^{1}$, Stephan Hofmann ${ }^{5}$, Luca \\ Camilli $^{1,4} *$ \\ 1: DTU Nanotech, Technical University of Denmark, 2800 Kgs. Lyngby, Denmark. \\ 2: SurfCat, Department of Physics, Technical University of Denmark, 2800 Kgs. Lyngby, Denmark. \\ 3: DTU Fotonik, Technical University of Denmark, Ørsteds Plads Building 343, DK-2800 Kongens Lyngby, Denmark \\ 4: Center for Nanostructured Graphene (CNG), Technical University of Denmark, Ørsteds Plads Building 345C, DK-2800 \\ Kongens Lyngby, Denmark \\ 5: Department of Engineering, University of Cambridge, Cambridge CB3 0FA, United Kingdom
}

KEYWORDS: hexagonal boron nitride, 2D materials, ellipsometry, thickness, chemical vapor deposition, wafer-scale mapping

\begin{abstract}
The availability of an accurate, non-destructive method for measuring thickness and continuity of 2D materials with monolayer sensitivity over large areas is of pivotal importance for the development of new applications based on two-dimensional (2D) materials. While simple optical contrast methods and electrical measurements are sufficient for the case of metallic and semiconducting 2D materials, the low optical contrast and high electrical resistivity of wide band gap dielectric 2D materials such as hexagonal boron nitride (hBN) hampers their characterization. In this work, we demonstrate a non-destructive method to quantitatively map the thickness and continuity of hBN monolayers and bilayers over large areas. The proposed method is based on acquisition and subsequent fitting of ellipsometry spectra of $\mathrm{hBN}$ on $\mathrm{Si} / \mathrm{SiO}_{2}$ substrates. Once a proper optical model is developed, it becomes possible to identify and map the commonly observed polymer residuals from the transfer process and obtain sub-monolayer thickness sensitivity for the hBN film. With some assumptions on the optical functions of hBN, the thickness of an as-transferred hBN monolayer on $\mathrm{SiO}_{2}$ is measured as $4.1 \AA \pm 0.1 \AA$, whereas the thickness of an air-annealed hBN monolayer on $\mathrm{SiO}_{2}$ is measured as $2.5 \AA \pm 0.1 \AA$. We argue that the difference in the two measured values is due to the presence of a water layer trapped between the $\mathrm{SiO}_{2}$ surface and the hBN layer in the latter case. The procedure can be fully automated to wafer-scale and extended to other 2D materials transferred onto any polished substrate, as long as their optical functions are approximately known.
\end{abstract}

\section{INTRODUCTION}

Wafer-scale realization of devices based on 2D materials and their heterostructures ${ }^{1}$ will depend on the availability of methods to characterize those materials quickly, reliably and non-destructively over large areas. Notably, two properties of 2D materials where one can envision stringent requirements from the industry are continuity and well-defined, homogeneous thickness.

Optical contrast and Raman spectroscopy are two of the most widely used methods to characterize thickness of 2D materials such as graphene and transition metal dichalcogenides. The optical contrast of those materials relative to a $\mathrm{SiO}_{2}$ substrate is often sufficient to distinguish the number of layers - down to monolayer thickness - by simple inspection with an optical microscope. ${ }^{2-4}$ Raman spectroscopy can also be used to determine the layer thickness of these systems, since features like peak position, intensity and broadening vary consistently with the layer numbers. ${ }^{5-7}$

On the other hand, thickness determination of wide band gap 2D materials such as hexagonal boron nitride $(\mathrm{hBN})$ is still an open issue, despite the key role of $\mathrm{hBN}$ in the realization of electronic and optoelectronic devices based on 2D 
materials. ${ }^{8-11}$ Due to its visible- and near-infrared transparency, the optical contrast of hBN is very low. Even if the thickness of the typical $\mathrm{SiO}_{2}$ thin film substrate is optimized to achieve maximum contrast in $\mathrm{hBN}$, the contrast remains below $2.5 \%$ for a hBN monolayer. ${ }^{12}$ Moreover, in the case of transferred chemical vapor deposited (CVD) $\mathrm{hBN}$, polymer residues will exacerbate this issue by strongly altering the overall optical contrast. The width and position of the main hBN Raman peak has been shown to depend on thickness in few-layer hBN, but the dependence is so weak that it is comparable to the standard deviation of the measurement from sample to sample. ${ }^{12,13}$ Furthermore, the intensity of the main hBN Raman peak is about 50 times smaller than the intensity of the $G$ peak of graphene under the same measurement conditions ${ }^{12}$, so that longer integration time is needed for the former case. Finally, coupling this last point with the fact that spot sizes for Raman measurements are typically of the order of $1 \mu \mathrm{m}$ means that wafer-scale analysis can be quite timeconsuming. The hBN photoluminescence peak position depends on thickness up to 100 layers but thickness differences of one layer are too small to be resolved. ${ }^{14}$

Unlike the case of electrically conductive 2D materials like graphene, the continuity of hBN cannot be measured by electrical probes ${ }^{15}$ due to its insulating nature. Alternative thickness characterization methods involving high vacuum equipment exist (e.g. transmission electron microscopy and scanning Auger spectroscopy) but they are small-scale and time-consuming. ${ }^{16}$ Atomic force microscopy (AFM) could also be used, but AFM is not suitable for rapid analysis over large areas, and the measurements can be affected by adsorbed water or polymer residues, in the case of transferred films. ${ }^{17}$ Therefore, a fast, reliable and non-destructive thickness characterization method scalable to large areas and complementary to the tools today available is highly desirable.

Spectroscopic ellipsometry is the industry-standard technique for mapping the thickness of thin-film materials on large scale. In fact, it has been applied before to determine the thickness and optical functions of $\mathrm{BN}$ films thicker than some tens of $\mathrm{nm}$ in amorphous, cubic, and hexagonal structure. $^{18-22}$ Applying spectroscopic ellipsometry to 2D materials is not straightforward and presents specific challenges, mostly due to the very small thicknesses involved. This means that thickness characterization by ellipsometry has so far been possible only for 2D materials (such as graphene ${ }^{22,23}$ and $\mathrm{MoS}_{2}{ }^{22,24}$ ) whose thickness can be confirmed easily and reliably by other methods. A recent publication related to the application of ellipsometry for thickness imaging in 2D materials ${ }^{22}$ showed thickness results on few-layers graphene and $\mathrm{MoS}_{2}$, followed by thickness results on much thicker $\mathrm{hBN}(>100 \mathrm{~nm}$ ). No studies on thickness determination of mono- or few-layer hBN by ellipsometry exist to our best knowledge, except for a brief mention with limited experimental details. ${ }^{25}$

In this work, we first identify the key steps in the sample preparation phase and in the data analysis phase that are necessary in order to obtain reliable results from ellipsometry measurements on 2D hBN. Then, we demonstrate the ability of the ellipsometry technique to map the thickness and continuity of CVD-grown hBN down to monolayer thickness over regions of about $1 \mathrm{~cm}^{2}$, which can in principle be extended to any arbitrary area.

\section{EXPERIMENTAL DETAILS}

\section{Process A}

Two sets of samples were used in this work. For the first set of samples (Process A), hBN was grown on commercially available platinum foil using a custom built low-pressure CVD furnace. The deposition follows an established process similar to growth of hBN on copper, which is discussed in detail elsewhere. ${ }^{26}$ Namely, the samples are heated to the growth temperature of $1100^{\circ} \mathrm{C}$ and exposed to borazine vapor at a pressure of $1 \times 10^{-5}$ for 5 minutes. After growth, the samples are transferred by semi-dry mechanical delamination onto a $\mathrm{Si}$ substrate pre-coated with $80 \mathrm{~nm} \mathrm{SiO}_{2}$ using a poly(vinyl alcohol) (PVA) stamp. In particular, since platinum interacts weakly with $2 \mathrm{D}$-materials ${ }^{27}$ it is possible to directly delaminate the $2 \mathrm{D}$ films by mechanical force. Therefore, a PVA layer is first coated onto the sample and then delaminated after drying. The PVA/h-BN stack can then be deposited directly on the target substrate, in case of monolayer transfer. If multiple layers are required, the same $\mathrm{PVA} / \mathrm{h}-\mathrm{BN}$ stack is deposited onto another as-grown h-BN/Pt sample, delaminated again and then deposited onto the target substrate. It is worth noting that in this process the second h$\mathrm{BN}$ layer only comes into contact with the first h-BN layer and thus there cannot be any polymer residues between the two hBN layers. The final step of the transfer process is the dissolution of the PVA in DI water.

To enable precise identification of positions on the substrate and measurement repeatability, a periodic array of metallic index marks was fabricated on the $\mathrm{Si} / \mathrm{SiO}_{2}$ substrate by photolithography, metal evaporation, and lift-off (see Fig. S1, Supporting Information).

\section{Process B}

For the second set of samples (Process B), hBN was grown on electropolished copper foil inside a commercial rapidthermal CVD system (AS-One, Annealsys). Details of the electropolishing procedure can be found elsewhere. ${ }^{28}$ For CVD growth, both the borazine vapor and the thermal energy coming from infrared lamps were pulsed in order to reduce the damage to the chamber due to the reaction with the infrared radiation sensitive borazine precursor. ${ }^{29}$ Details can be found in the Supporting Information. hBN was then transferred to a $\mathrm{Si} / \mathrm{SiO}_{2}$ wafer with index marks (as in Process A) by a standard wet etching transfer procedure using a spin-coated poly(methyl methacrylate) (PMMA) film as a handling layer. ${ }^{30}$ Notably, the $\mathrm{Cu}$ substrate was etched in a $5 \mathrm{wt} \% \mathrm{HCl}$ solution containing $0.5 \mathrm{wt} \%$ hydrogen peroxide. The PMMA film used as handling layer was removed in acetone after transfer to the $\mathrm{SiO}_{2}$ target substrate. However, even after $24 \mathrm{~h}$ in acetone, we found it impossible 
to obtain reliable ellipsometry results on samples fabricated by Process $\mathrm{B}$ due to large amounts of residual PMMA contamination. Therefore, those samples were annealed in air at $300^{\circ} \mathrm{C}$. By a combination of optical contrast and ellipsometry, it was found that most of the residual PMMA had burned off during the annealing step, while leaving the hBN layer intact (Fig. S2, Supporting Information). Unless otherwise specified, all characterization of samples fabricated by Process B (including ellipsometry measurements) was performed after the annealing step in air.

Spectroscopic ellipsometry is an optical technique for determining the thickness and optical functions of one or more stacked thin film materials. Incident white light containing a broad spectral range of photon energies $E$ with known $p$ - and s-polarization components is reflected by the films and their substrate. The overall reflection coefficients of the sample at each photon energy for the two polarization components depend both on the optical functions of the film(s) and substrate (that is, refractive index $n(E)$ and extinction coefficient $k(E)$ ) and on their thicknesses due to interference effects. Reflected light is detected by the ellipsometer, which measures the magnitude, $\tan (\Psi(E))$, and phase, $\Delta(E)$, of the ratio between the reflection coefficients for the $p$ - and s-polarization components. Hence, any unknown film thickness and optical functions in the sample can in principle be traced back by fitting the $\Psi$ and $\Delta$ spectra to a layered optical model where those unknown quantities are the fitting parameters. We used a rotating compensator spectroscopic ellipsometer (M-2000, J.A. Woollam Co.) with a measurement spot size of approximately 200 x $400 \mu \mathrm{m}$ (Fig. S1, Supporting Information). Thickness inhomogeneity on a smaller scale than the spot size is therefore not resolved with the current measurement configuration. To a first approximation, the thickness measured on an inhomogeneous spot will be a weighted average of the thickness distribution within that spot. If spatial resolution is the main concern, spectroscopic imaging ellipsometry could in principle be employed. ${ }^{22}$ The measurement of $\Psi(E)$ and $\Delta(E)$ at each measured spot was taken at two angles of incidence $\vartheta\left(60^{\circ}\right.$ and $\left.70^{\circ}\right)$ in the photon energy range 0.7-5.9 eV. By repeating the ellipsometry measurement on different spots, the $\Psi$ and $\Delta$ spectra were mapped automatically over a region of the order of $1 \mathrm{~cm}^{2}$ depending on the specific sample. The distance between the center of any adjacent measurement was 500 $\mu \mathrm{m}$, so that there is a gap of $100 \mu \mathrm{m}$ between each adjacent measurement. With the above acquisition parameters, which are not optimized for speed, the measurement time is 90 $\mathrm{min} / \mathrm{cm}^{2}$. If measurement time is the main concern, singlewavelength imaging ellipsometry could be an alternative option, even though the thickness sensitivity may decrease. The program CompleteEase, version 5.06 (J.A. Woollam Co.) was used to define possible optical models for the investigated layer structure, to fit the $\Psi$ and $\Delta$ spectra against those models, and to determine the mean square error (MSE) of the fit, as well as the error bars of the fitted parameters and their correlation coefficients (Figs. S3-S5, Supporting Information). Once the optical model is defined, it takes about $2 \mathrm{~min}$ to fit an area of $1 \mathrm{~cm}^{2}$ without any user interaction and using only a standard laptop computer.

Analysis of the position, intensity, and width of the main Raman peak of hBN (1366-1370 $\mathrm{cm}^{-1}$ ) was used to doublecheck the thickness measured by ellipsometry at different locations across the samples. ${ }^{12}$ We used a Raman system with a $532 \mathrm{~nm}$ laser and a 50x objective lens (Renishaw). Note that the spot size of the Raman measurement is about 1 $\mu \mathrm{m}$, i.e., two orders of magnitude smaller than the ellipsometry spot size. For this reason, more than 100 Raman measurements over a $100 \times 100 \mu \mathrm{m}$ grid were performed at each location. The presence of hBN on the sample was further determined by $\mathrm{x}$-ray photoemission spectroscopy (XPS, Thermo Scientific K-Alpha) with a monochromatized $\mathrm{Al} \mathrm{K} \mathrm{K}_{\alpha} \mathrm{X}$-ray source and nominal spot size of $400 \mu \mathrm{m}$. The $1 \mathrm{~s}$ core levels of $\mathrm{B}, \mathrm{N}$, and $\mathrm{C}$ were used to quantify atomic composition with analysis depth of a few nm. Optical maps were acquired using a Nikon Eclipse L200D microscope equipped with a programmable Prior Scientific XYZ stage. Background subtraction from individual optical images and subsequent stitching of images to form an optical map was conducted as described elsewhere. ${ }^{4}$ Scanning electron microscopy (SEM) characterization was performed with a Zeiss Supra 40 VP SEM. Atomic force microscopy (AFM) experiments were carried out in air in semi-contact mode with a NTEGRA AFM from NT-MDT Spectrum Instruments.

\section{OPTICAL MODELING}

Although spectroscopic ellipsometry appears to be an ideal technique for characterizing thickness and optical functions of $2 \mathrm{D}$ materials, a number of possible issues must be dealt with in order to obtain reliable results. Here we briefly introduce those issues.

Substrate: An area of the $\mathrm{Si} / \mathrm{SiO}_{2}$ substrate that is not covered by $\mathrm{hBN}$ is used as a reference ellipsometry measurement. This is used to determine the thickness and optical functions of the $\mathrm{SiO}_{2}$ layer, which are then regarded as known quantities when measuring on the full $\mathrm{Si} / \mathrm{SiO}_{2} / \mathrm{hBN}$ stack, thus minimizing the number of fitting parameters. We find that the thickness of the thermally-grown $\mathrm{SiO}_{2}$ is relatively uniform, with standard deviation of $0.6 \AA$ across a $0.5 \mathrm{~cm}^{2}$ region mapped with 228 equally spaced measurement points (Fig. S5, Supporting Information).

Ambiguity between thickness determination and optical function determination: A general limitation of the ellipsometry technique for very thin layers $(<10 \mathrm{~nm})$ is that changes in $\Psi$ and $\Delta$ spectra due to thickness variations are indistinguishable from changes in $\Psi$ and $\Delta$ spectra due to optical function variations. ${ }^{31,32}$ It follows that either the thickness or the optical functions of hBN must be treated as a known parameter when fitting ellipsometry spectra. The procedure followed in this study is to test a set of optical functions from a given literature source and fit the hBN 

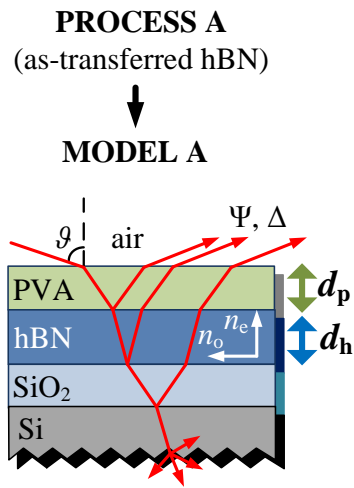

(a)
PROCESS B

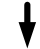

MODEL B

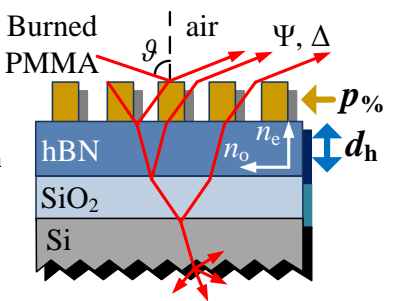

(b) (post-annealed hBN)

Figure 1. Optical models used to interpret $\Psi$ and $\Delta$ spectra in this work. The quantities in bold are the fitting parameters. (a) Model A, used for the samples fabricated with Process A. The fitting parameters are the hBN thickness $\left(d_{\mathrm{h}}\right)$ and the PVA thickness $\left(d_{\mathrm{p}}\right)$. (b) Model B, used for the samples fabricated with Process B. The fitting parameters are the hBN thickness $\left(d_{\mathrm{h}}\right)$ and the fraction of surface covered by burned PMMA ( $\left.p_{\%}\right)$

thickness treating the optical functions as known. The procedure is then repeated with another set of optical functions from another literature source to assess the sensitivity of the fitted thickness on the details of the optical functions.

Optical function modeling and optical anisotropy: hBN is uniaxially negative, that is, the ordinary refractive index $n_{o}$ in the $a b$ plane of the material is larger than the extraordinary refractive index $n_{e}$ along its $c$ axis (Fig. S6, Supporting Information). ${ }^{19,33}$ Therefore, two different refractive index spectra must be specified for hBN when defining its optical functions in the ellipsometry model: one in the plane of the substrate $\left(n_{o}\right)$ and one along the growth direction $\left(n_{e}\right)$, as shown in Fig. 1. There are two main references for the refractive index of hBN that rigorously took anisotropy into account and are used in this work: Schubert et al. ${ }^{19}$ and Ishii et al. $^{33}$ They are reproduced in Fig. S6, Supporting Information. Even though we will compare thickness results using the refractive index sets from both references, Schubert's reference values are our preferred choice because they were measured (i) on thin films instead of on single crystals, (ii) by ellipsometry, and (iii) more recently. Since the band gap of hBN is expected to lie slightly outside our measured spectral range, ${ }^{34}$ the extinction coefficient $k$ is assumed to be zero over the whole range.

Polymer contamination: For the case of 2D materials grown by CVD, an additional complication for ellipsometry measurements is the use of a polymer support layer in the transfer process. Although the polymer is chemically removed after transfer by use of suitable solvents, some residuals are inevitably observed on top of the transferred material. ${ }^{35}$ Clearly, the ellipsometry spectra $\Psi(E)$ and $\Delta(E)$ can be influenced by the thickness and optical functions of the residual polymer (Fig. 1). If the residual polymer is not optically modeled when fitting ellipsometry spectra, the thickness of the 2D material can be grossly overestimated (Fig. S2, Supporting Information). In fact, the only difference between the optical models used for Process A and for Process B is the treatment of polymer contamination. In Process A, the transfer polymer (PVA) covered the stamp completely during transfer. Therefore, a continuous PVA layer above the hBN layer is assumed in Model A (Fig. 1(a)). Its thickness is a fitted parameter $\left(d_{\mathrm{p}}\right)$, whereas its optical functions are taken from the literature ${ }^{36}$ and kept fixed. In Process $\mathrm{B}$, the transfer polymer (PMMA) was burned off after transfer. Therefore, a discontinuous PMMA layer above the hBN layer is assumed in Model B (Fig. 1(b)). Its thickness $(10 \mathrm{~nm})$ is estimated by AFM measurements (Fig. S15, Supporting Information) and kept fixed, whereas its surface coverage is a fitted parameter $\left(p_{\%}\right)$. The optical functions of PMMA are altered by air annealing, and they are therefore estimated by a separate ellipsometry measurement on burned PMMA and kept fixed (Fig. S8, Supporting Information).

Angular spread of light beam: In order to obtain a relatively small spot size in the ellipsometry measurement, the incident light beam is focused by a lens. If the resulting angular spread of the incident beam ( $7^{\circ}$ in our setup) is not modeled in the data fitting phase, the fitted hBN thickness can be significantly different and often inconsistent with Raman results. A significantly lower MSE of the fit (6.3 versus 9.8) is also achieved when the angular spread effect is included.

Once the above precautions have been observed, the MSE of the fit and the correlation coefficient between fitted parameters become sufficiently low to (i) obtain submonolayer thickness sensitivity, and to (ii) distinguish between hBN and polymer contamination (Figs. S3-S4, Supporting Information).

\section{RESULTS}

\section{Process A}

Samples fabricated with Process A were modeled as shown in Fig. 1(a). Nominally, a continuous hBN layer with fixed, anisotropic optical functions (either those by Schubert et al. ${ }^{19}$ or those by Ishii et al. ${ }^{33}$ ) lies beneath a continuous PVA layer. The only two fitting parameters are the hBN thickness $\left(d_{\mathrm{h}}\right)$ and the PVA thickness $\left(d_{\mathrm{p}}\right)$. Results from two samples fabricated following Process A are shown in Fig. 2. Sample 1 is a monolayer of $\mathrm{hBN}$, while a transfer of two monolayers of hBN was attempted for Sample 2. An optical map of Sample 1 (Fig. 2(a)) appears uniform with some polymer contamination easily visible in the top of the map. A scratch (from the transfer process) through the center of Sample 2 is easily visible, while a darker and possibly thicker hBN region is seen in the region surrounding the small cross on the map. From optical microscopy it is thus possible to visualize hBN layers, but the low contrast of the hBN mixed with possible polymer contamination altering the optical response makes it practically impossible to implement automated optical coverage analysis as done for graphene. ${ }^{4} \mathrm{~A}$ comparison of the ellipsometric hBN thickness maps (Fig. 2(c,d)) with optical maps (Fig. 2(a,b)) reveals that the areas where hBN was transferred are correctly detected by the 

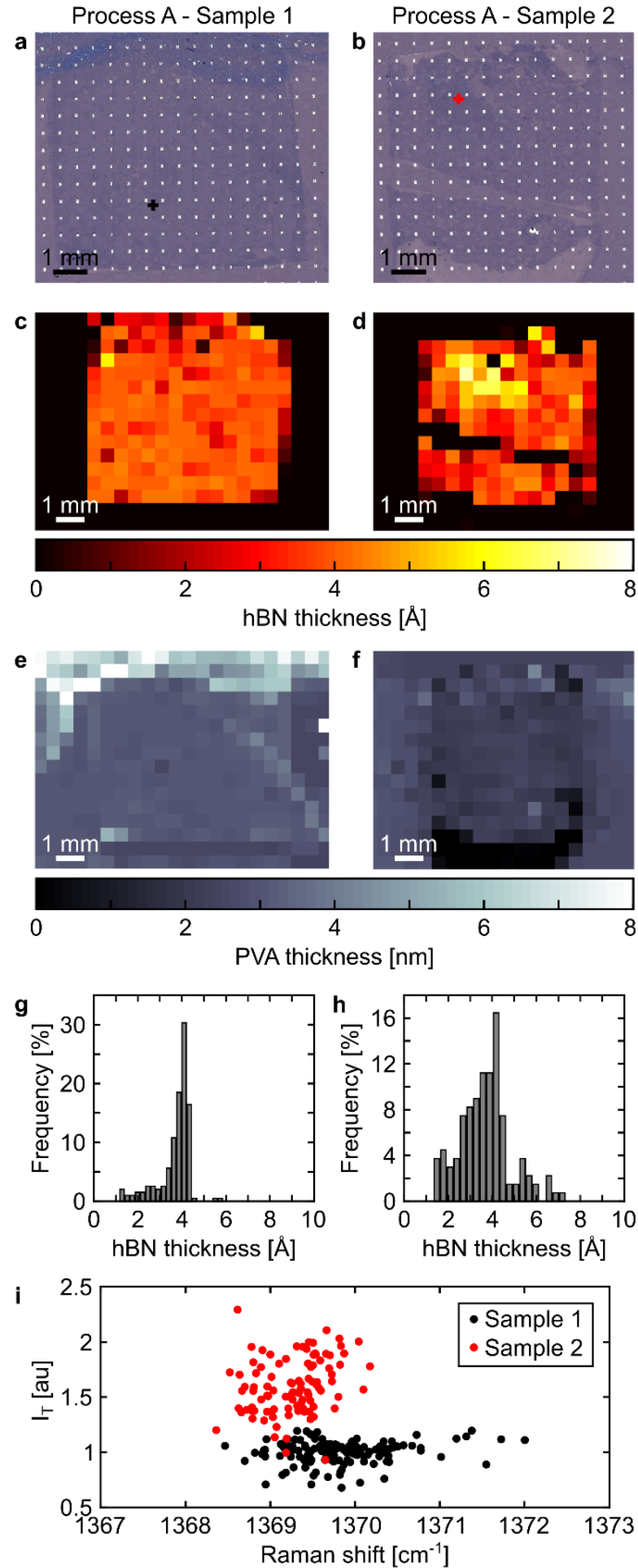

Figure 2. Characterization of samples fabricated by Process A. (a,b) Optical maps of Sample 1 and Sample 2. Contrast has been enhanced ( $\sim 80 \%)$ to increase visibility of hBN layers. The small crosses highlight positions for Raman maps. (c,d) Ellipsometric hBN thickness maps (fitting parameter $d_{\mathrm{h}}$ ) of Sample 1 and Sample 2 with corresponding histograms of hBN thickness in (g,h). The hBN optical functions measured by Schubert et al. are used to obtain the ellipsometry maps. (e,f) Ellipsometric thickness maps of polymer (PVA) layer (fitting parameter $d_{\mathrm{p}}$ ) in Sample 1 and Sample 2. (i) Scatter plot of integrated Raman peak intensity $\left(\mathrm{I}_{\mathrm{T}}\right)$ and Raman peak position for Sample 1 and Sample 2.
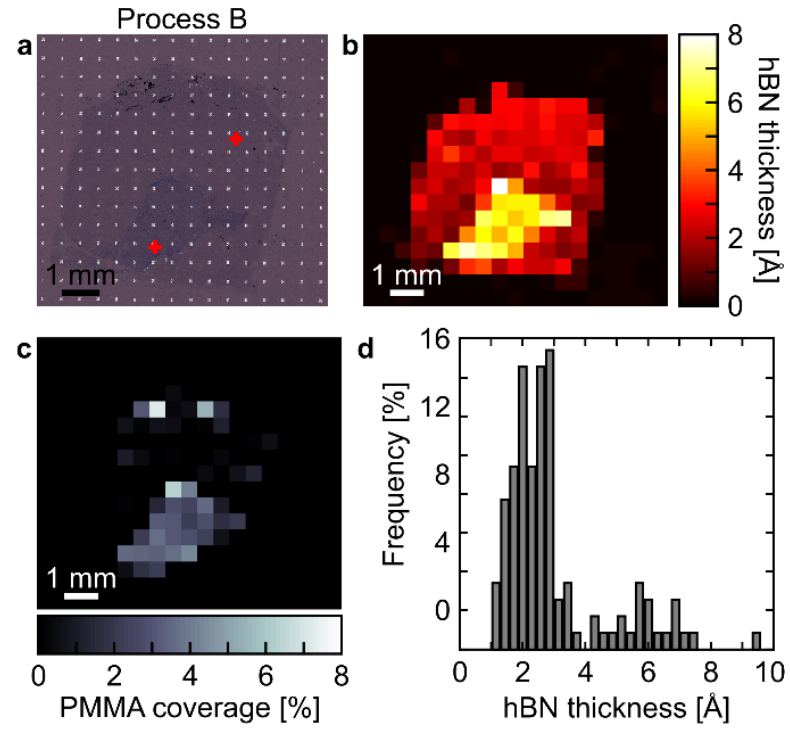

Figure 3. Characterization of sample fabricated by Process B. (a) Optical map. The small crosses highlight positions for Raman characterization shown in Fig. S10, Supporting Information. (b) Ellipsometric hBN thickness map (fitting parameter $d_{\mathrm{h}}$ ) of the sample in (a) with corresponding histogram of hBN thickness in (d). The hBN optical functions measured by Schubert et al. are used to obtain the ellipsometry map. (c) Ellipsometric map of polymer (PMMA) coverage in the mixed PMMA/air top layer (fitting parameter $\left.p_{\%}\right)$.

ellipsometry measurement. In particular, the sample discontinuity caused by a scratch and visible in the optical map of Fig. 2(b) is promptly picked up by the ellipsometry map and attributed to a discontinuity in the hBN layer (Fig. 2(d)) rather than in the polymer layer (Fig. 2(f)). The brighter area at the bottom of the optical map of Sample 2 (Fig. 2(b)) is attributed to the absence of both $\mathrm{hBN}$ and of the polymer contamination layer. This highlights the applicability of ellipsometry measurements for extracting the thickness of hBN. From the optical map the scratch could easily be viewed as a single layer of hBN compared to the bare $\mathrm{SiO}_{2}$ region, while the actual single layer hBN region would then optically be detected as a bilayer region. Finally, the dark area visible at the top of the optical map of Sample 1 (Fig. 2(a)) is correctly attributed to polymer contamination (Fig. 2(e)).

Sample 1, which was fabricated aiming for monolayer hBN, appears to have a fairly uniform thickness over the whole sample area according to ellipsometry (Fig. 2(c)). Analysis of the thickness distribution statistics using Schubert's optical functions for hBN (Fig. 2(e)) yields a thickness count peaking around $4.1 \AA$. When Ishii's optical functions are employed, the thickness distribution retains its shape but is shifted to lower thicknesses, with the count peak appearing at 2.4 Å thickness instead (Fig. S9, Supporting Information). Given that the interplanar spacing of bulk hBN along its caxis is $3.33 \AA$, and that a layer of adsorbed water may be present between $\mathrm{SiO}_{2}$ and $\mathrm{hBN},{ }^{23}$ the peak in measured thickness is likely to correspond to a monolayer of hBN on $\mathrm{SiO}_{2}$. The thickness difference between the two optical 
function models can be ascribed to the higher (roughly 10\%) refractive index measured by Ishii et al., which implies that light propagation in $\mathrm{hBN}$ is slower than in the case of Schubert's refractive index, and therefore a lower hBN thickness is necessary to obtain the same phase shift for light reflected at the $\mathrm{Si} / \mathrm{SiO}_{2}$ interface and propagating twice through the hBN layer. Note that, regardless of the assumed optical functions, the thickness distribution has a tail for thicknesses below the peak thickness, whereas it decays rapidly for thicknesses above the peak thickness. This is expected for a sample that is never thicker than a hBN monolayer, but that may be discontinuous in some areas. Indeed, if the sample is not continuous over the area of a single measurement, the resulting thickness will be smaller than the value for a continuous monolayer sheet, as we have discussed earlier, in the Experimental Details section.

Sample 2 exhibits a broader thickness distribution in the ellipsometry map (Fig. 2(d,f)). It peaks at a similar thickness (4.1 $\AA$ ) as the previous sample, but it has a longer tail in both directions. This might be due to the fact that measurements in a transition region between two and one layer will give a number that is between these two thicknesses. Nevertheless, from the ellipsometry map it is straightforward to pinpoint the location of a bilayer area (Fig. 2(d)). Raman spectroscopy, performed at pixels with measured thickness above $6 \AA$ and around $4 \AA$ (Fig.2(g)), confirms indeed the presence of bilayer and monolayer $h B N$, respectively. We find a red shift of $0.5 \mathrm{~cm}^{-1}$ in the hBN Raman peak position and an increase in the integrated intensity, both of which correspond to the differences between mono- and bilayer hBN reported elsewhere. ${ }^{13}$ For a fairly inhomogeneous specimen like Sample 2, ellipsometry is a particularly useful technique because the relative thickness accuracy between different spots is only limited by the standard error of the fit, which is less than $0.1 \AA$ in this study (Fig. S3, Supporting Information).

\section{Process B}

Since the samples fabricated with Process B were subjected to air annealing in order to remove most of the polymer contamination, a separate optical model (Model B) was developed for those samples, as shown in Fig. 1(b). The hBN layer itself is modeled exactly as in Model A. However, the PMMA optical functions are modified to account for the fact that the few PMMA leftovers have been burned and therefore exhibit absorption in the visible. The modified PMMA optical

functions are still kept fixed from point to point as in Model A. Since only scattered PMMA leftovers are expected after air annealing (Fig. S2, Supporting Information), the PMMA layer is modeled as a mix of air and PMMA (Fig. 1(b)). The PMMA coverage in the mixed layer is a fitting parameter $\left(p_{\%}\right)$, whereas its thickness $d_{\mathrm{p}}$ is a fixed parameter (more details available in the Supporting Information).

Optical and ellipsometric thickness maps of a sample fabricated with Process B and air-annealed are shown in Fig. 3(a,b). Interestingly, two well-separated areas of significantly different thicknesses are revealed, which results in a double peak in the corresponding thickness distribution (Fig. 3(d)). The first peak has the most counts around a thickness of 2.5 $\AA$. The second peak has the most counts at around 6.0 A. The second peak matches well with the sum of the thickness of the first peak plus the interplanar spacing of bulk hBN (that is, $2.5 \AA+3.3 \AA=5.8 \AA$ ). Raman spectroscopy also suggests from an increase in the integrated peak area that the pixels of $6 \AA$ thickness are bilayer areas, whereas the pixels of $2.5 \AA$ thickness are monolayer areas (Fig. S10, Supporting Information). The same trend is qualitatively confirmed by the higher intensity of XPS B 1s and N 1s core level peaks in the areas identified as bilayers (Figs. S11-S12, Supporting Information). Interestingly, a higher PMMA coverage is detected in the areas with thicker hBN (Fig. 3(c)), which is also confirmed by the higher intensity of the $\mathrm{C} 1 \mathrm{~s}$ core level peak in the XPS spectra related to those areas (Figs. S12S13, Supporting Information) and by SEM and AFM images (Figs. S14-S15 in Supplementary Information). If not correctly detected, this would lead to overestimation of the hBN thickness by optical techniques. For example, the region with thicker hBN could be incorrectly identified as a trilayer due to the additional contrast from the PMMA. Distinguishing between the hBN layer and polymer residues is therefore of great importance also for post-annealed samples fabricated by Process B.

Finally, we note that there is a considerable difference between Process A and Process $\mathrm{B}$ regarding the thickness of a hBN monolayer (4.1 $\AA$ versus $2.5 \AA$ ). This difference can be explained by the likely presence of an adsorbed water layer between $\mathrm{SiO}_{2}$ and $\mathrm{hBN}$ on as-transferred samples (Process A), similar to the case of graphene. ${ }^{37,38}$ Such a water interlayer may be expelled upon annealing at $300^{\circ} \mathrm{C}$ as in Process B causing the hBN to conform better to the substrate similarly to graphene. ${ }^{39}$ Since water layers are not included in our ellipsometry model to keep the model complexity low, it is then possible that the $4.1 \AA$ thickness of a hBN monolayer, as measured in the samples fabricated with Process A, includes the thickness of a water layer.

\section{CONCLUSION}

Through multi-angle spectroscopic ellipsometry, the thickness and continuity of two-dimensional hBN grown by CVD and transferred to a $\mathrm{Si} / \mathrm{SiO}_{2}$ substrate was mapped over a centimeter squared area. For the first time, sub-monolayer thickness sensitivity was demonstrated for hBN grown by different CVD processes, with or without post-transfer annealing. Once the presence of polymer leftovers from the transfer process was appropriately included in the optical model used to fit ellipsometry spectra, the thickness of hBN could be determined down to a monolayer, consistently with Raman and XPS analysis. Assuming that the hBN optical functions employed in this study are correct, the thickness of an as-transferred hBN monolayer on $\mathrm{SiO}_{2}$ is measured as 4.1 $\AA \pm 0.1 \AA$, whereas the thickness of an air-annealed hBN monolayer on $\mathrm{SiO}_{2}$ is measured as $2.5 \AA \pm 0.1 \AA$. The discrepancy is likely due to the removal of a water interlayer between hBN and $\mathrm{SiO}_{2}$ upon air annealing. In hBN bilayers, 
the spacing between the first and second layer is roughly 3.3 $\AA$, consistently with the interplanar spacing of bulk hBN. Due to its single layer sensitivity and its ability to distinguish between hBN and polymer contamination, ellipsometry appears as a more reliable tool for thickness identification compared to optical contrast methods, especially if coupled to SEM or AFM techniques for the characterization of morphology of polymer residues in the case of transferred films. The fast, non-destructive large-area mapping capabilities demonstrated here for the case of hBN may be extended to other 2D materials whose thickness is too difficult or too slow to characterize with other techniques. Extending the method to other materials requires, however, that reasonable guesses for their optical functions and for the characteristics of any transfer-related polymer contamination are available.

\section{ASSOCIATED CONTENT}

\section{Supporting Information.}

See the Supporting Information for comparison of the measured and fitted ellipsometry spectra, typical mean squared errors of the fits, preliminary ellipsometry measurements on the bare substrate, the two sets of reference hBN optical functions - as well as the optical functions of the PVA and PMMA layers - used for fitting ellipsometry spectra, the consequences of post-transfer annealing on the thickness maps, the difference in the estimated hBN thickness with different assumed hBN optical functions, complementary Raman and XPS measurements confirming the ellipsometry results, further hBN synthesis details, further details on the mixed PMMA/air layer employed to fit ellipsometry spectra, and SEM and AFM investigations to characterize the density and morphology of polymer residues in the samples fabricated through Process B.

\section{AUTHOR INFORMATION}

\section{CORRESPONDING AUTHOR}

*ancro@fysik.dtu.dk, lcam@nanotech.dtu.dk

\section{AUTHOR CONTRIBUTIONS}

The manuscript was written through contributions of all authors. All authors have given approval to the final version of the manuscript.

\section{FUNDING SOURCES}

A.C. acknowledges funding from the Innovation Fund Denmark (65733), from the Danish Council for Strategic Research (12-132644), and from Villum Fonden (9455). P.R.W. and L.C. acknowledge funding from the Danish National Research Foundation (DNRF) Center for Nanostructured Graphene (DNRF103). P.R.W. also acknowledge funding from EU Graphene Flagship Core 1 (696656). L.C. acknowledges funding by the Young Investigator Program of Villum Fonden, project no. 19130. S.H. and R.W. acknowledge funding by EPSRC (EP/K016636/1).

\section{REFERENCES}

(1) Kang, K.; Lee, K. H.; Han, Y.; Gao, H.; Xie, S.; Muller, D. A.; Park, J. Layer-by-Layer Assembly of Two-Dimensional Materials into Wafer-Scale Heterostructures. Nature 2017, 550 (7675), 229233.

(2) Blake, P.; Hill, E. W.; Castro Neto, A. H.; Novoselov, K. S.; Jiang, D.; Yang, R.; Booth, T. J.; Geim, A. K. Making Graphene Visible. Appl. Phys. Lett. 2007, 91 (6), 63124.

(3) Li, H.; Wu, J.; Huang, X.; Lu, G.; Yang, J.; Lu, X.; Xiong, Q.; Zhang, H. Rapid and Reliable Thickness Identification of Two-Dimensional Nanosheets Using Optical Microscopy. ACS Nano 2013, 7 (11), 10344-10353.

(4) Jessen, B. S.; Whelan, P. R.; Mackenzie, D. M. A.; Luo, B.; Thomsen, J. D.; Gammelgaard, L.; Booth, T. J.; Bøggild, P. Quantitative Optical Mapping of Two-Dimensional Materials. Sci. Rep. 2018, 8 (1), 6381.

(5) Ferrari, A. C.; Meyer, J. C.; Scardaci, V.; Casiraghi, C.; Lazzeri, M.; Mauri, F.; Piscanec, S.; Jiang, D.; Novoselov, K. S.; Roth, S.; Geim, A. K. Raman Spectrum of Graphene and Graphene Layers. Phys. Rev. Lett. 2006, 97 (18), 187401.

(6) Li, H.; Zhang, Q.; Yap, C. C. R.; Tay, B. K.; Edwin, T. H. T.; Olivier, A.; Baillargeat, D. From Bulk to Monolayer MoS2: Evolution of Raman Scattering. Adv. Funct. Mater. 2012, 22 (7), 1385-1390.

(7) Berkdemir, A.; Gutiérrez, H. R.; Botello-Méndez, A. R.; Perea-López, N.; Elías, A. L.; Chia, C.-I.; Wang, B.; Crespi, V. H.; López-Urías, F.; Charlier, J.-C.; Terrones, U.; Terrones, M. Identification of Individual and Few Layers of WS2 Using Raman Spectroscopy. Sci. Rep. 2013, 3 (1), 1755.

(8) Dean, C. R.; Young, A. F.; Meric, I.; Lee, C.; Wang, L.; Sorgenfrei, S.; Watanabe, K.; Taniguchi, T.; Kim, P.; Shepard, K. L.; Hone, J. Boron Nitride Substrates for High-Quality Graphene Electronics. Nat. Nanotechnol. 2010, 5 (10), 722-726.

Lundeberg, M. B.; Gao, Y.; Woessner, A.; Tan, C.; Alonso-González, P.; Watanabe, K.; Taniguchi, T.; Hone, J.; Hillenbrand, R.; Koppens, F. H. L. Thermoelectric Detection and Imaging of Propagating Graphene Plasmons. Nat. Mater. 2017, 16 (2), 204-207.

(10) Shiue, R.-J.; Gao, Y.; Wang, Y.; Peng, C.; Robertson, A. D.; Efetov, D. K.; Assefa, S.; Koppens, F. H. L.; Hone, J.; Englund, D. HighResponsivity Graphene-Boron Nitride Photodetector and Autocorrelator in a Silicon Photonic Integrated Circuit. Nano Lett. 2015, 15 (11), 7288-7293.

(11) Lee, G.-H.; Yu, Y.-J.; Cui, X.; Petrone, N.; Lee, C.H.; Choi, M. S.; Lee, D.-Y.; Lee, C.; Yoo, W. J.; Watanabe, K.; Tanigushi, T.; Nuckolls, C.; Kim, P.; Hone, J. Flexible and Transparent MoS 2 FieldEffect Transistors on Hexagonal Boron NitrideGraphene Heterostructures. ACS Nano 2013, 7 (9), 7931-7936.

(12) Gorbachev, R. V.; Riaz, I.; Nair, R. R.; Jalil, R.; Britnell, L.; Belle, B. D.; Hill, E. W.; Novoselov, K. S.; Watanabe, K.; Taniguchi, T.; Geim, A. K.; Blake, P. Hunting for Monolayer Boron Nitride: Optical and Raman Signatures. Small 2011, 7 (4), 465-468.

(13) Cai, Q.; Scullion, D.; Falin, A.; Watanabe, K.; 
Taniguchi, T.; Chen, Y.; Santos, E. J. G.; Li, L. H. Raman Signature and Phonon Dispersion of Atomically Thin Boron Nitride. Nanoscale 2017, 9 (9), 3059-3067.

(14) Du, X. Z.; Uddin, M. R.; Li, J.; Lin, J. Y.; Jiang, H. $\mathrm{X}$. Layer Number Dependent Optical Properties of Multilayer Hexagonal BN Epilayers. Appl. Phys. Lett. 2017, 110 (9), 3-6.

(15) Buron, J. D.; Pizzocchero, F.; Jessen, B. S.; Booth, T. J.; Nielsen, P. F.; Hansen, O.; Hilke, M.; Whiteway, E.; Jepsen, P. U.; Bøggild, P.; Petersen, D. Electrically Continuous Graphene from Single Crystal Copper Verified by Terahertz Conductance Spectroscopy and Micro Four-Point Probe. Nano Lett. 2014, 14 (11), 6348-6355.

(16) Sutter, P.; Sutter, E. Thickness Determination of Few-Layer Hexagonal Boron Nitride Films by Scanning Electron Microscopy and Auger Electron Spectroscopy. APL Mater. 2014, 2 (9), 92502.

(17) Cheng, Z.; Zhou, Q.; Wang, C.; Li, Q.; Wang, C.; Fang, Y. Toward Intrinsic Graphene Surfaces: A Systematic Study on Thermal Annealing and WetChemical Treatment of SiO 2 -Supported Graphene Devices. Nano Lett. 2011, 11 (2), 767-771.

(18) Ren, S. L.; Rao, A. M.; Eklund, P. C.; Doll, G. L. Ellipsometric Study of Boron Nitride Thin-film Growth on Si(100). Appl. Phys. Lett. 1993, 62 (15), 1760-1762.

(19) Schubert, M.; Rheinländer, B.; Franke, E.; Neumann, H.; Hahn, J.; Röder, M.; Richter, F. Anisotropy of Boron Nitride Thin-Film Reflectivity Spectra by Generalized Ellipsometry. Appl. Phys. Lett. 1997, 70 (14), 1819.

(20) Franke, E.; Schubert, M.; Hecht, J.-D.; Neumann, H.; Tiwald, T. E.; Thompson, D. W.; Yao, H.; Woollam, J. A.; Hahn, J. In Situ Infrared and Visible-Light Ellipsometric Investigations of Boron Nitride Thin Films at Elevated Temperatures. J. Appl. Phys. 1998, 84 (1), 526-532.

(21) Zapien, J. A.; Messier, R.; Collins, R. W. Ultraviolet-Extended Real-Time Spectroscopic Ellipsometry for Characterization of Phase Evolution in BN Thin Films. Appl. Phys. Lett. 2001, 78 (14), 1982-1984.

(22) Funke, S.; Wurstbauer, U.; Miller, B.; Matković, A.; Green, A.; Diebold, A.; Röling, C.; Thiesen, P. H. Spectroscopic Imaging Ellipsometry for Automated Search of Flakes of Mono- and N-Layers of 2DMaterials. Appl. Surf. Sci. 2017, 421, 435-439.

(23) Ochoa-Martínez, E.; Gabás, M.; Barrutia, L.; Pesquera, A.; Centeno, A.; Palanco, S.; Zurutuza, A.; Algora, C. Determination of a Refractive Index and an Extinction Coefficient of Standard Production of CVD-Graphene. Nanoscale 2015, 7 (4), 1491-1500.

(24) Yim, C.; O’Brien, M.; McEvoy, N.; Winters, S.; Mirza, I.; Lunney, J. G.; Duesberg, G. S. Investigation of the Optical Properties of MoS2 Thin Films Using Spectroscopic Ellipsometry. Appl. Phys. Lett. 2014, 104 (10), 103114.

(25) Cho, Y.; Summerfield, A.; Davies, A.; Cheng, T. S.; Smith, E. F.; Mellor, C. J.; Khlobystov, A. N.; Foxon, C. T.; Eaves, L.; Beton, P. H.; Novikov, S. V. Hexagonal Boron Nitride Tunnel Barriers Grown on Graphite by High Temperature Molecular Beam Epitaxy. Sci. Rep. 2016, 6 (1), 34474.

(26) Kidambi, P. R.; Blume, R.; Kling, J.; Wagner, J. B.; Baehtz, C.; Weatherup, R. S.; Schloegl, R.; Bayer, B. C.; Hofmann, S. In Situ Observations during Chemical Vapor Deposition of Hexagonal Boron Nitride on Polycrystalline Copper. Chem. Mater. 2014, 26 (22), 6380-6392.

(27) Weatherup, R. S.; D’Arsié, L.; Cabrero-Vilatela, A.; Caneva, S.; Blume, R.; Robertson, J.; Schloegl, R.; Hofmann, S. Long-Term Passivation of Strongly Interacting Metals with Single-Layer Graphene. J. Am. Chem. Soc. 2015, 137 (45), 14358-14366.

(28) Yu, F.; Camilli, L.; Wang, T.; Mackenzie, D. M. A.; Curioni, M.; Akid, R.; Bøggild, P. Complete LongTerm Corrosion Protection with Chemical Vapor Deposited Graphene. Carbon N. Y. 2018, 132, 7884.

(29) Stoot, A.; Camilli, L. Chemical Vapour Deposition from a Radiation-Sensitive Precursor. WO2017153510, 2017.

(30) Li, X.; Zhu, Y.; Cai, W.; Borysiak, M.; Han, B.; Chen, D.; Piner, R. D.; Colombo, L.; Ruoff, R. S. Transfer of Large-Area Graphene Films for HighPerformance Transparent Conductive Electrodes. Nano Lett. 2009, 9 (12), 4359-4363.

(31) Archer, R. J. Determination of the Properties of Films on Silicon by the Method of Ellipsometry. $J$. Opt. Soc. Am. 1962, 52 (9), 970.

(32) Jellison, G. E. Data Analysis for Spectroscopic Ellipsometry. In Handbook of Ellipsometry; Tompkins, H. G., Irene, E. A., Eds.; Springer, 2005.

(33) Ishii, T.; Sato, T. Growth of Single Crystals of Hexagonal Boron Nitride. J. Cryst. Growth 1983, 61 (3), 689-690.

(34) Watanabe, K.; Taniguchi, T.; Niiyama, T.; Miya, K.; Taniguchi, M. Far-Ultraviolet Plane-Emission Handheld Device Based on Hexagonal Boron Nitride. Nat. Photonics 2009, 3 (10), 591-594.

(35) Hallam, T.; Berner, N. C.; Yim, C.; Duesberg, G. S. Strain, Bubbles, Dirt, and Folds: A Study of Graphene Polymer-Assisted Transfer. Adv. Mater. Interfaces 2014, 1 (6), 1400115.

(36) Schnepf, M. J.; Mayer, M.; Kuttner, C.; Tebbe, M.; Wolf, D.; Dulle, M.; Altantzis, T.; Formanek, P.; Förster, S.; Bals, S.; König, T. A. F.; Fery, A. Nanorattles with Tailored Electric Field Enhancement. Nanoscale 2017, 9 (27), 9376-9385.

(37) Bom, N. M.; Soares, G. V.; De Oliveira Junior, M. H.; Lopes, J. M. J.; Riechert, H.; Radtke, C. Water Incorporation in Graphene Transferred onto $\mathrm{SiO} 2 / \mathrm{Si}$ Investigated by Isotopic Labeling. J. Phys. Chem. C 2016, 120 (1), 201-206.

(38) Lee, M. J.; Choi, J. S.; Kim, J.-S.; Byun, I.-S.; Lee, D. H.; Ryu, S.; Lee, C.; Park, B. H. Characteristics and Effects of Diffused Water between Graphene and a SiO2 Substrate. Nano Res. 2012, 5 (10), 710717.

(39) Gammelgaard, L.; Caridad, J. M.; Cagliani, A.; Mackenzie, D. M. A.; Petersen, D. H.; Booth, T. J.; Bøggild, P. Graphene Transport Properties upon Exposure to PMMA Processing and Heat Treatments. 2D Mater. 2014, 1 (3), 35005. 


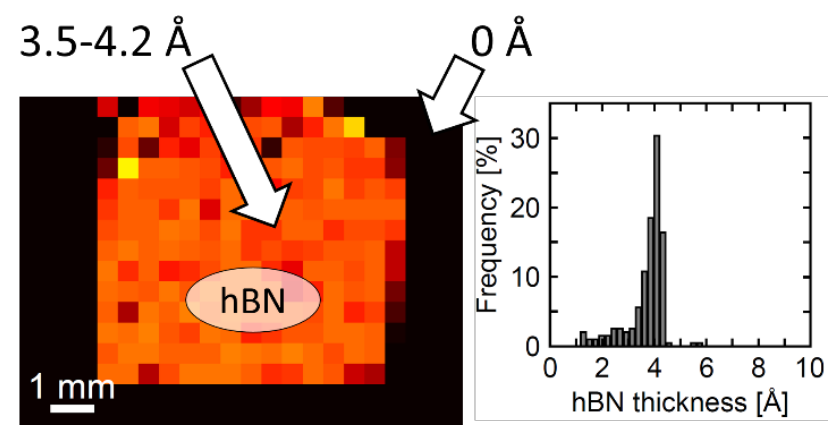

